\begin{tabular}{|l|r|}
\hline Recebido em: $18 / 05 / 2006$ & HOLOS Environment, v.7 n.1, 2007 - P. 1 \\
\hline Liberado para Publicação em: 18/01/2007 & ISSN:1519-8421 (CD-ROM) / ISSN:1519-8634 (ON-LINE) \\
\hline
\end{tabular}

\title{
PERFIL DA QUALIDADE DAS ÁGUAS SUBTERRANEAS DE MANAUS
}

\section{PROFILE OF THE QUALITY OF MANAUS CITY'S GROUNDWATERS}

\author{
Silva, M. L ${ }^{1}$; Silva, M. S. R. ${ }^{2}$ \\ ${ }^{1}$ Instituto Nacional de Pesquisa da Amazônia - INPA \\ Av. André Araújo, N 1756, Aleixo, 69060-000 - Manaus (AM), Fone (092) 643- \\ 3153, e-mail: marciols@inpa.gov.br \\ ${ }^{2}$ Instituto Nacional de Pesquisa da Amazônia - INPA \\ Av. André Araújo, N 1756, Aleixo, 69060-000 - Manaus (AM), Fone (092) 643- \\ 3165, e-mail: ssilva@inpa.gov.br
}

\section{RESUMO}

Manaus, capital do Estado do Amazonas, está localizada próxima ao centro da Bacia Amazônica à margem esquerda do rio Negro e sobre os sedimentos terciários areno-argilosos da Formação Alter do Chão, de idade cretácea. A região está associada a um índice médio pluviométrico de $2.300 \mathrm{~mm} / \mathrm{ano}$, um clima quente e úmido e uma exuberante cobertura vegetal. $\mathrm{O}$ abastecimento de água de Manaus é realizado por empresa particular, através de distribuição a partir de poços tubulares e por captação da drenagem superficial do rio Negro. Objetivando caracterizar e avaliar a qualidade das águas subterrâneas de Manaus, durante o período de cheia, aferiu-se as variáveis físicas, físico-químicas e químicas das amostras de águas de poços tubulares selecionados. Os resultados obtidos mostraram que as águas estudadas têm concentrações baixas de constituintes dissolvidos com pequeno acréscimo nos teores dos elementos, indicando rápida infiltração das águas superficiais e que são excelentes para consumo humano e industrial, por situarem-se dentro dos limites de potabilidade estabelecidos pela legislação vigente.

Palavras-chave: Hidroquímica. Contaminação. Potabilidade. Amazonas.

\begin{abstract}
Manaus city, capital of the Amazonas state, is at the left bank of Rio Negro. The surrounding region is characterized by the geological Alter do Chão Formation
\end{abstract}


consisting of sandy-clay tertiary sediments. An important annual rainfall of 2,300 $\mathrm{mm}$, classifies the climate as hot humid with a highly divers vegetation. The city water supply is mainly provided by tubular wells whose recharge comes from rain and Rio Negro water. A private enterprise explores captation and public water supply. The ground water of Manaus was evaluated during the rainy period. Tubular wells water are selected and water sampled for physical and physic-chemical analysis. The obtained data pointed out that the waters have low dissolved constituent concentrations with a low increase of the elements in the rainy period. They also indicated rapid infiltration, of surface water and excellent human and industrial consumption quality. The water quality is in concordance with standards established in current legislation.

Key words: Hydrochemistry. Contamination. Water quality. Amazonas.

\section{INTRODUÇÃO}

A cidade de Manaus, com população de 1.403.796 habitantes, representando 49,4\% da população do estado do Amazonas (IBGE, 2002), encontra-se a $03^{\circ} 08^{\prime}$ $30^{\prime}$ 'S e $60^{\circ} 00^{\prime} 00^{\prime \prime} \mathrm{W}$ à altitude de $21 \mathrm{~m}$ acima do nível do mar e localiza-se à margem esquerda do rio Negro (Figura 1), próximo de sua confluência com o rio Solimões.

O clima de Manaus é do tipo Ami de monções, segundo a classificação de Köppen. A temperatura média anual é de $25,6^{\circ} \mathrm{C}$, com amplitude térmica de $4^{\circ} \mathrm{C}$. O índice pluviométrico médio anual é de 2.000 a $2.300 \mathrm{~mm}$. A umidade do ar é alta, em torno de $88 \%$ no período chuvoso e $77 \%$ no seco (PRANCE e LOVEJOY, 1985).

A área de estudo envolve os sedimentos continentais da Formação Alter do Chão, constituída por sedimentos vermelhos sob a forma de argilas (cor vermelha mosqueada, cinza-esbranquiçada, branca-avermelhada, plástica, maciços, às vezes finamente laminados com filmes de areia, com predominância de minerais do grupo da caulinita associada a traços de gibbsita, feldspatos, quartzo e raramente illita), argilitos (com lentes de areia, pouco consolidados e cor vermelha, creme, branca e roxo), siltitos, arenitos (finos a médios, geralmente com estratificação cruzada e intercalações argilosas, micáceos, ferruginosos, maciços e bioturbados) e conglomerados (com seixos de quartzo), pobres em fósseis, e recoberta discordantemente, em parte, por sedimentos terciários ou diretamente por sedimentos quaternários (DAMIÃO et al., 1972; PETRI e FÚLFARO, 1988; CUNHA et al., 1994).

O Holoceno está representado por depósitos aluvionares ao longo dos rios e igarapés. Os lateritos têm ampla distribuição e boa representatividade, formados pelos horizontes transicional, argiloso, ferruginoso, esferolítico, linha de pedra e solo (PETRI e FÚLFARO, 1988; FERNANDES FILHO et al., 1997). 


\begin{tabular}{|l|r|}
\hline Recebido em: $18 / 05 / 2006$ & HOLOS Environment, v.7 n.1, 2007 - P. 3 \\
\hline Liberado para Publicação em: 18/01/2007 & ISSN:1519-8421 (CD-ROM) / ISSN:1519-8634 (ON-LINE) \\
\hline
\end{tabular}

Os sistemas de aqüíferos Arenito Alter do Chão e Barreiras, na bacia sedimentar do Amazonas, têm um volume de água igual a $32.500 \mathrm{~km}^{3}$ e poços com intervalo de vazão de 10 a $400 \mathrm{~m}^{3} / \mathrm{h}$ (REBOUÇAS, 1997).

A partir de 1979, a Companhia de Pesquisa de Recursos Minerais (CPRM) começou a perfurar poços no município de Manaus. Estes com profundidade máxima em torno de 220 metros, todos explorando aqüíferos constituídos pela Formação Alter do Chão, de idade cretácea (DAEMON, 1975), que sob Manaus, apresentam-se geralmente como aqǘf́eros não confinados. Na área urbana, o abastecimento de água é realizado por empresa particular, sendo efetuado através de poços tubulares e captação da drenagem superficial do rio Negro.

Nos últimos anos, vem crescendo a utilização dos mananciais subterrâneos no Estado do Amazonas, por ser de fácil disponibilidade, boa qualidade e custo relativamente baixo.

Assim, este trabalho tem por objetivo, fornecer informações sobre o comportamento hidroquímico das águas subterrâneas de alguns poços da cidade de Manaus, em período de cheia, e caracterizar agentes poluidores antropogênicos que possam estar atingindo a área.

\section{MATERIAL E MÉTODOS}

As coletas de amostras foram realizadas no mês de maio (cheia local) visando comparar os dados obtidos por Silva (1999) em período seco. Foram amostrados quatorze poços distribuídos no perímetro urbano de Manaus, selecionados em função da localização, acesso e se estavam em funcionamento (Figura 1). 


\begin{tabular}{|l|r|}
\hline Recebido em: $18 / 05 / 2006$ & HOLOS Environment, v.7 n.1, 2007 - P. 4 \\
\hline Liberado para Publicação em: 18/01/2007 & ISSN:1519-8421 (CD-ROM) / ISSN:1519-8634 (ON-LINE) \\
\hline
\end{tabular}

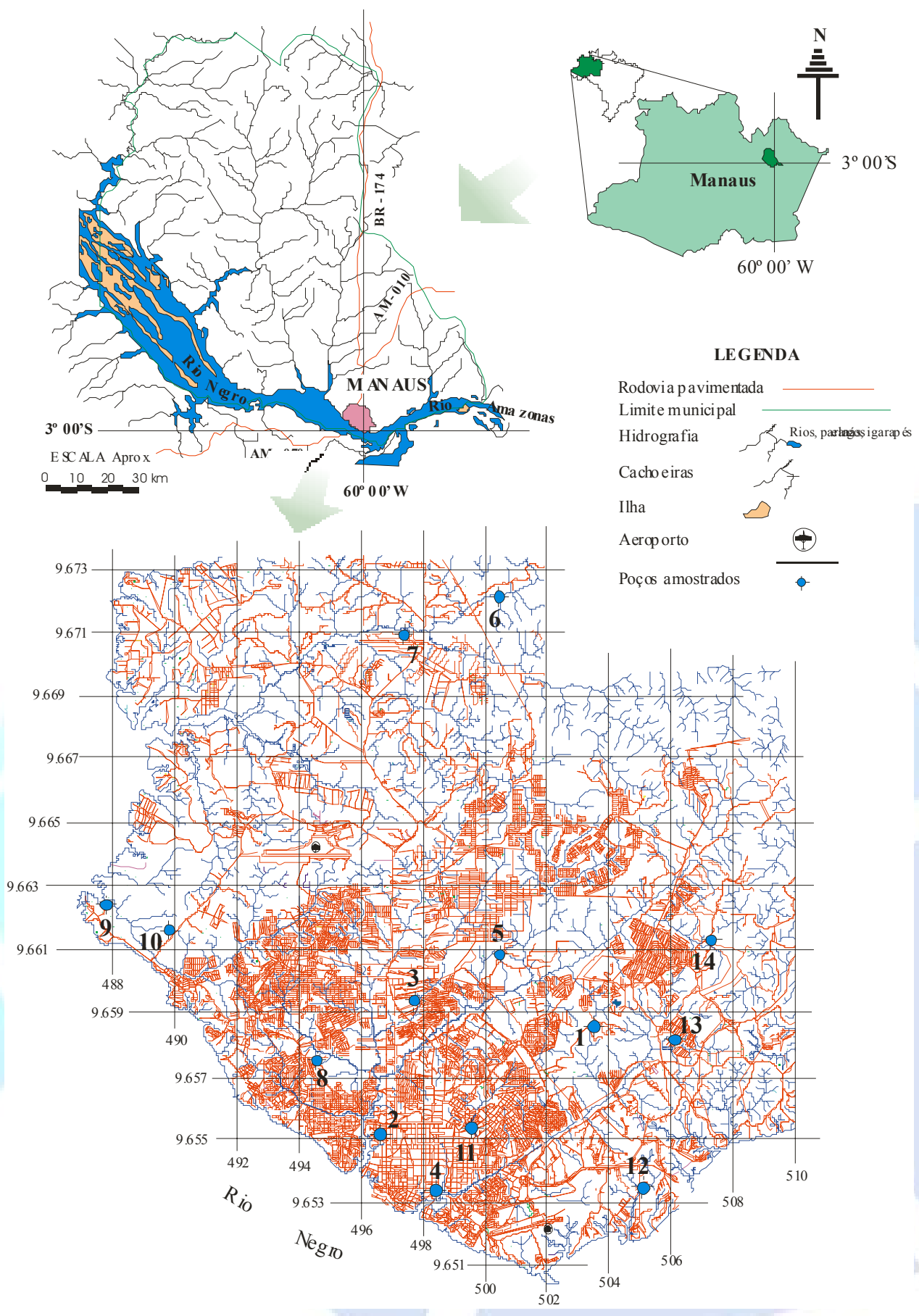

Figura 1. Mapa de localização da cidade de Manaus - AM e poços amostrados.

Depois de determinadas às temperaturas, as amostras foram levadas imediatamente ao Instituto Nacional de Pesquisa da Amazônia - INPA, para serem aferidos $\mathrm{pH}$, condutividade elétrica, turbidez e variáveis químicas selecionadas, segundo as metodologias resumidamente apresentadas no Quadro 1. 
Quadro 1 - Metodologias para Determinação Hidroquímica das Águas Subterrâneas Amostradas em Manaus -AM

\begin{tabular}{|c|c|c|c|}
\hline Variável & Método* & Autores & Unidade \\
\hline $\begin{array}{c}\text { Cálcio }\left(\mathrm{Ca}^{2+}\right), \text { Magnésio } \\
\left(\mathrm{Mg}^{2+}\right) \text { e Dureza }\end{array}$ & Complexometria EDTA & Golterman et al. (1978) & $\mathrm{mg} \cdot \mathrm{L}^{-1}$ \\
\hline Potássio $\left(\mathrm{K}^{+}\right)$e Sódio $\left(\mathrm{Na}^{+}\right)$ & Fotometria de chama & MICRONAL, modelo - B262 & $\operatorname{mg} . \mathrm{L}^{-1}$ \\
\hline $\begin{array}{l}\text { Ferro total e Ferro dissolvido } \\
\qquad\left(\mathrm{Fe}^{3+}\right)\end{array}$ & APHA & APHA (1985) & $\mathrm{mg} \cdot \mathrm{L}^{-1}$ \\
\hline Alcalinidade & $\begin{array}{l}\text { Potenciometria - } \\
\text { titulante } \mathrm{HCl} 0,05 \mathrm{~N} \text { até }\end{array}$ & Golterman et al. (1978) & $\mathrm{mgHCO}_{3}^{-} \cdot \mathrm{L}^{-1}$ \\
\hline & $\mathrm{pH} 4,3$ & & \\
\hline Cloreto $\left(\mathrm{Cl}^{-}\right)$ & Titulometria & Golterman et al. (1978) & $\mathrm{mg} \cdot \mathrm{L}^{-1}$ \\
\hline Fósfato $\left(\mathrm{PO}_{4}\right)$ & Espectrofotometria & Golterman et al. (1978) & $\mathrm{mg} \cdot \mathrm{L}^{-1}$ \\
\hline \multirow[t]{3}{*}{ Nitrato $\left(\mathrm{NO}_{3}^{-}\right)$} & $\begin{array}{c}\text { Redução a } \mathrm{NO}_{2} \\
\text { Redução por cádmio }\end{array}$ & Strickland and Parsons (1968) & $\mathrm{mg} \cdot \mathrm{L}^{-1}$ \\
\hline & Adaptada ao método & & \\
\hline & FIA & & \\
\hline \multirow[t]{2}{*}{ Amônio $\left(\mathrm{NH}_{4}^{+}\right)$} & Método de Nessler & Strickland and Parsons (1968) & $\operatorname{mg} . \mathrm{L}^{-1}$ \\
\hline & $\begin{array}{c}\text { Adaptada ao método } \\
\text { FIA }\end{array}$ & & \\
\hline Sulfato $\left(\mathrm{SO}_{4}^{-}\right)$ & Turbidimétrico & APHA (1985) & $\mathrm{mg} \cdot \mathrm{L}^{-1}$ \\
\hline Silica reativa $\left(\mathrm{Si}(\mathrm{OH})_{4}\right)$ & Colorimetria & Golterman et al. (1978) & $\mathrm{mg} \cdot \mathrm{L}^{-1}$ \\
\hline
\end{tabular}

*APHA (American Public Health Association); FIA (Sistema de Injeção em Fluxo); EDTA (ácido etilenodiamino tetracético).

\section{RESULTADOS E DISCUSSÃO}

De acordo com as características hidrogeológicas, os poços amostrados extraem água da Formação Alter do Chão. Os perfis litológicos mostram aqüíferos livres e semiconfinados por camadas de argila e pelo Arenito Manaus (Tabela 1).

A altitude relacionada aos poços amostrados variou de $29,00 \mathrm{~m}$ no poço $\mathrm{n}^{\circ} 9$ a $110,00 \mathrm{~m}$ no poço $\mathrm{n}^{\circ} 6$. A média para o nível estático foi de $41,65 \mathrm{~m}$ e nível dinâmico de $68,80 \mathrm{~m}$. As profundidades médias dos poços e filtros foram de $132,71 \mathrm{~m}$ e 87,2 $\mathrm{m}$, respectivamente, com cimentação média de 63,82 m (Tabela 1). 


\begin{tabular}{|l|r|}
\hline Recebido em: $18 / 05 / 2006$ & HOLOS Environment, v.7 n.1, 2007 - P. 6 \\
\hline Liberado para Publicação em: $18 / 01 / 2007$ & ISSN:1519-8421 (CD-ROM) / ISSN:1519-8634 (ON-LINE) \\
\hline
\end{tabular}

Os valores obtidos para a temperatura do ar e das águas subterrâneas foram relativamente homogêneos, com médias de $31,1^{\circ} \mathrm{C}$ e $28,6^{\circ} \mathrm{C}$, respectivamente (Tabela 2). Silva e Bonotto (2000) e Silva (1999) registraram temperaturas médias das águas subterrâneas em Manaus, no período de seca, de $27,8^{\circ} \mathrm{C}$. Silva (2005) verificou que para Manaus, a correlação linear entre as temperaturas das águas de subsuperfície e as temperaturas do ar, nos períodos de coletas, foi de $83,20 \%$. Indicando, assim, rápida influência da temperatura atmosférica nessas águas estudadas.

Quanto à hidroquímica, o poço $\mathrm{n}^{\mathrm{o}} 11$ apresentou valores anômalos quando comparados às médias estudadas, sendo assim, excluído do trato estatístico. Os valores de pH variaram de 4,2 a 5,7 (Tabela 2), indicando que são ácidas e não se enquadram nos padrões de qualidade ambiental (portaria 518/2004 do Ministério da Saúde), pois, são inferiores ao limite mínimo de $\mathrm{pH}$ igual a 6,5 , o que pode ser corrigido por tamponamento no tratamento dessas águas. $\mathrm{O}$ não enquadramento dentro dos padrões recomendados, não a descaracteriza como água potável, já que o $\mathrm{pH}$ está relacionado à geologia da região, cobertura vegetal, recarga rápida e processo de interação água-rocha/solo.

Os valores encontrados são semelhantes aos de Forti e Moreira-Nordemann (1991) de 4 a 5,3, em água de chuva na região e Silva e Bonotto (2000) de 4,1 a 5,4, em águas de poços tubulares em Manaus. Essa característica é função da pobreza química dos materiais percolados, cobertura vegetal e recarga rápida.

Os dados apresentados na tabela 2 mostram variação de condutividade elétrica (CE), de $11,10 \mu \mathrm{S} . \mathrm{cm}^{-1}$ a $57,7 \mu \mathrm{S} . \mathrm{cm}^{-1}$ (média de 32,19 $\mu \mathrm{S} . \mathrm{cm}^{-1}$ ), tendo correlação de $96 \%$ com K $\mathrm{K}^{+}, 89 \%$ com $\mathrm{pH}, 84 \%$ com profundidade dos filtros, $75 \%$ com $\mathrm{Ca}^{2+} \mathrm{e} \mathrm{Na}^{+}$, $70 \%$ com bicarbonato e $67 \%$ com $\mathrm{Mg}^{2+}$. Silva (2005) verificou, em Manaus, correlações da $\mathrm{CE}$ com o $\mathrm{Mg}^{2+}(71,78 \%), \mathrm{Ca}^{2+}(69,18 \%), \mathrm{Cl}^{-}(66,30 \%), \mathrm{NO}_{3}{ }^{-}$ $(65,67 \%)$. Estas correlações indicam a importância dessas variáveis químicas para a condutividade elétrica do meio estudado no período estudado.

$\mathrm{O}$ argilo mineral dominante nos solos da região é a caulinita (DAMIÃO et al., 1972), indicando condições da existência de silicatos de alumínio que fixam o magnésio. O que explica a correlação do magnésio de $92 \%$ com o bicarbonato, $81 \%$ com $\mathrm{pH}$ e $75 \%$ com a profundidade dos filtros.

Em abundância, considerando os valores médios obtidos para os principais cátions, verifica-se a seqüência $\mathrm{K}^{+}>\mathrm{Ca}^{2+}>\mathrm{Na}^{+}>\mathrm{Mg}^{2+}$. Moraes e Costa (1997) ao estudarem o caulim de Manaus-Itacoatiara verificaram a seguinte seqüência de cátions: $\mathrm{Mg}^{2+}>\mathrm{Ca}^{2+}>\mathrm{K}^{+}>\mathrm{Na}^{+}$, enquanto Silva (1999) estudando águas subterrâneas de Manaus verificou a seqüência $\mathrm{K}^{+}>\mathrm{Na}^{+}>\mathrm{Ca}^{2+}>\mathrm{Mg}^{2+}$.

O ganho de potássio é, provavelmente, resultado do intemperismo químico do substrato rochoso, pois apresenta correlação de $89 \%$ com $\mathrm{pH}, 72 \%$ com a profundidade dos filtros e $-0,61 \%$ e $-0,61 \%$ com o nível freático em período de cheia e seca (SILVA, 1999), respectivamente. 


\begin{tabular}{|l|r|}
\hline Recebido em: $18 / 05 / 2006$ & HOLOS Environment, v.7 n.1, 2007 - P. 7 \\
\hline Liberado para Publicação em: 18/01/2007 & ISSN:1519-8421 (CD-ROM) / ISSN:1519-8634 (ON-LINE) \\
\hline
\end{tabular}

Tabela 1-Características Hidrogeológicas dos Poços Amostrados em Manaus-AM

\begin{tabular}{|c|c|c|c|c|c|c|c|c|c|}
\hline \multirow[t]{2}{*}{ Amostra } & \multicolumn{2}{|c|}{$\begin{array}{l}\text { Localização } \\
\text { (UTM) }\end{array}$} & \multirow{2}{*}{$\begin{array}{l}\text { Alt. } \\
\mathrm{m}\end{array}$} & \multirow{2}{*}{$\begin{array}{l}\text { N.E. }^{2} \\
\mathrm{~m}\end{array}$} & \multirow{2}{*}{$\begin{array}{l}\text { N.D. } \\
\mathrm{m}\end{array}$} & \multirow{2}{*}{$\begin{array}{l}\text { Prof. }^{4} \\
\text { M }\end{array}$} & \multirow{2}{*}{$\begin{array}{c}\text { Filtro }^{5} \\
\mathrm{~m}\end{array}$} & \multirow{2}{*}{$\begin{array}{c}\text { Cim. }^{6} \\
\mathrm{~m}\end{array}$} & \multirow[t]{2}{*}{ Litológia* $^{*}$ e Prof. ${ }^{4}$} \\
\hline & Leste & Norte & & & & & & & \\
\hline 1 & 503137 & 9657483 & 87,00 & 22,92 & 44,33 & 143,00 & 58,5 & 40,00 & $\begin{array}{c}\text { 0-Ag-6-Ar-21-Ag-26-Ar-50-Ag-56-Ar-71- } \\
\text { Ag-88-Ar-102-Ag-105-Ar-118-Ag-123- } \\
\text { Ar-143 }\end{array}$ \\
\hline 2 & 496760 & 9654436 & 52,00 & 37,60 & 46,47 & 168,00 & 148,0 & 120,00 & $\begin{array}{c}\text { 0-Ag-3-Ar-9-Ag-18-Ar-24-Ag-33-Ar-36- } \\
\text { Ag-93-Ar-129-Ag-144-Ar-168 }\end{array}$ \\
\hline 3 & 498200 & 9658100 & 50,00 & 47,40 & 57,00 & 120,00 & 82,0 & 70,00 & $\begin{array}{c}\text { 0-Ag-26-ArM-40-Ag-50-Ar-54-Ag-66-Ar- } \\
\text { 72-Ag-88-Ar-86-Ag-94-Ar-121 }\end{array}$ \\
\hline 4 & 498500 & 9654181 & 35,00 & 29,50 & 90,00 & 168,00 & 158,0 & 130,00 & $\begin{array}{c}\text { 0-Ar-6-Ag-26-Ar-30-Ag-106-Ar-144-Ag- } \\
\text { 158-Ar-164-Ag-168 }\end{array}$ \\
\hline 5 & 500150 & 9659800 & 49,00 & 35,63 & 42,00 & 82,00 & 60,0 & 54,00 & $\begin{array}{c}\text { 0-S-3-Ag-15-Ar-21-Ag-24-Ar-42-ArC-45- } \\
\text { Ag-60-Ar-72-Ag-78-Ar-82 }\end{array}$ \\
\hline 6 & 500356 & 9667528 & 110,0 & 103,25 & 161,50 & 228,00 & 164,0 & 85,00 & $\begin{array}{c}\text { 0-Ag-66-Ar-96-Ag-132-Ar-186-Ag-198- } \\
\text { Ar-228 }\end{array}$ \\
\hline 7 & 496500 & 9666900 & 75,00 & 35,50 & 46,10 & 120,00 & 76,0 & 40,00 & $0-A g-18-A r-120$ \\
\hline 8 & 494426 & 9656260 & 50,00 & 34,15 & 51,38 & 80,00 & 64,0 & 54,00 & $0-\mathrm{S}-4-\mathrm{Ar}-15-\mathrm{Ag}-18-\mathrm{Ar}-80$ \\
\hline 9 & 489097 & 9661240 & 29,00 & 11,00 & 25,00 & 80,00 & 20,0 & 14,52 & $\begin{array}{c}\text { 0-Ar-14,02-ArM-14,52-ArC-32,68-Ag- } \\
\text { 46,09-Ar-51,26-Ag-54,70-ArM-58,70-Ag- } \\
\text { 67,61-ArM-68,11-Ar-74,59-Ag-75,57-Ar- } \\
\text { 78,57-Ag-89,50 }\end{array}$ \\
\hline 10 & 490205 & 9660769 & 35,00 & 14,05 & 38,50 & 66,00 & 52,0 & 50,00 & $\begin{array}{l}\text { 0-Ar-4-Ag-16-Ar-28-Ag-38-Ar-40-Ag-44- } \\
\text { Ar-48-Ag-52-Ar-54-Ag-62-Ar-66-ArM-70 }\end{array}$ \\
\hline 11 & 499183 & 9654985 & 49,00 & 22,00 & 36,37 & 77,00 & 64,0 & 30,00 & 0-Ag-6-Ar-36-Ag-42-Ar-75-Ag-77 \\
\hline 12 & 504477 & 9653797 & 65,00 & 55,00 & 104,50 & 138,00 & 60,0 & 50,00 & $\begin{array}{c}\text { 0-Ag-20-Ar-104-Ag-108-ArM-112-Ag- } \\
\text { 126-Ar-138 }\end{array}$ \\
\hline 13 & 505766 & 9656906 & 74,00 & 62,80 & 105,13 & 160,00 & 140,0 & 138,00 & $\begin{array}{c}\text { 0-Ag-10-Ar-56-Ag-82-Ar-104-ArM-110- } \\
\text { Ag-120-Ar-138-Ag-140-Ar-162 }\end{array}$ \\
\hline 14 & 506437 & 9660228 & 60,00 & 72,30 & 114,85 & 228,00 & 75,5 & 18,00 & $\begin{array}{c}\text { 0-Ag-7-Ar-92-ArM-94-Ar-100-Ag-103- } \\
\text { Ar-139-ArM-141-Ar-175-Ag-178-Ar-215- } \\
\text { Ag-228 }\end{array}$ \\
\hline
\end{tabular}

*Ar - areia, arenito; ArM- arenito Manaus; ArC - areia conglomerática; Ag - argila, argilito; S solo. ${ }^{1}$ Altirtude; ${ }^{2}$ Nível Estático; ${ }^{3}$ Nível Dinâmico; ${ }^{4}$ Profundidade; ${ }^{5}$ Filtro; ${ }^{6}$ Cimentação.

Os resultados de dureza variaram de $1,55 \mathrm{mg} . \mathrm{L}^{-1}$ a $15,26 \mathrm{mg} \cdot \mathrm{L}^{-1}$, tendo como média 6,29 mg. $\mathrm{L}^{-1}$ (Tabela 2). Houve aumento de acordo com o acréscimo da profundidade dos poços (correlação de $92 \%$ ). De acordo com a classificação de Sawyer e McCarty (1967), as águas subterrâneas estudadas são do tipo "moles", sendo esta classificação idêntica à verificada por Silva (1999).

Para as amostras analisadas, o teor de $\mathrm{Fe}^{3+}$ variou de $<0,005 \mathrm{mg} \cdot \mathrm{L}^{-1}$ a 0,441 $\mathrm{mg} . \mathrm{L}^{-1} \mathrm{e}$ o ferro total de $<0,005 \mathrm{mg} . \mathrm{L}^{-1}$ a $0,959 \mathrm{mg} . \mathrm{L}^{-1} \mathrm{e}$ média igual a $0,242 \mathrm{mg} . \mathrm{L}^{-1} \mathrm{e}$ 
0,127 mg. $\mathrm{L}^{-1}$, respectivamente (Tabela 2). A correlação deste íon com a turbidez foi de $99 \%$.

Em todas as amostras, o teor de sulfato foi inferior ao limite de detecção correspondente a $0,1 \mathrm{mg} . \mathrm{L}^{-1}$ enquanto que em termos de valores médios, o cloreto corresponde ao segundo ânion mais abundante (média de 1,04 mg. $\mathrm{L}^{-1}$ ) (Tabela 2).

$\mathrm{O}$ fosfato variou de $0,001 \mathrm{mg} \cdot \mathrm{L}^{-1}$ a $0,508 \mathrm{mg} \cdot \mathrm{L}^{-1}$, tendo como média 0,043 $\mathrm{mg} . \mathrm{L}^{-1}$. Esses valores são normais em águas naturais, pois o fosfato apresenta tendência de formar compostos com vários íons e coligações fortes com os minerais de argila.

O maior valor de bicarbonato das águas amostradas é igual a $39,65 \mathrm{mg} . \mathrm{L}^{-1}$ (Tabela 2) tendo correlação de $77 \%$ com a profundidade dos filtros. Para todos os poços, os valores estão bem abaixo do limite mínimo de potabilidade utilizado no Brasil.

Silva (1999) verificou a seguinte seqüência para os ânions: $\mathrm{Cl}^{-}>\mathrm{HCO}_{3}>\mathrm{SO}_{4}{ }^{2-}$ em águas subterrâneas de Manaus no período seco. Diferente $\left(\mathrm{HCO}_{3}>\mathrm{Cl}^{-}>\mathrm{SO}_{4}{ }^{2-}\right)$ do encontrado por Silva (1996) em águas de igarapés da mesma região, onde se verifica a similaridade com a seqüência para os teores deste estudo $\left(\mathrm{HCO}_{3}>\mathrm{Cl}^{-}>\mathrm{SO}_{4}{ }^{2-}\right)$ e o conseqüente ganho de bicarbonato no período de cheia. Constatou-se também o aumento dos teores de cloreto e sódio no período de seca e respectiva diminuição no período de cheia.

Os resultados referentes à amônia (Tabela 2) mostram homogeneidade e baixos teores para todas as amostras. $\mathrm{O}$ teor de nitrato nas amostras estudadas variou de $<0,010 \mathrm{mg} . \mathrm{L}^{-1}$ a $0,191 \mathrm{mg} . \mathrm{L}^{-1}$, tendo como média $0,073 \mathrm{mg} . \mathrm{L}^{-1}$ (Tabela 2). Em concentrações superiores a $10 \mathrm{mg} . \mathrm{L}^{-1}$, estabelecidos pela Organização Mundial de Saúde (OMS), são indesejáveis na água de uso doméstico, devido a um possível efeito tóxico sobre as crianças novas, causando metemoglobinemia ou doença azul. Em relação às águas coletadas por Silva (1999) no período de seca local, houve um pequeno aumento no teor médio de amônia e nitrato. $\mathrm{O}$ que se explica pelo não confinamento do aqüífero e rápida infiltração das águas superficiais.

O silício, embora seja o elemento mais largamente encontrado nas rochas, não ocorre em quantidades significativas nas águas subterrâneas. O intemperismo de minerais de argila e os feldspatos constituem as principais fontes desse elemento para as águas de subsuperfície. Teores de $25 \mathrm{mg} . \mathrm{L}^{-1}$ nas águas subterrâneas não são raros (SINELLI, 1979; SANTOS, 2000).

A concentração de sílica variou de $0,78 \mathrm{mg} \cdot \mathrm{L}^{-1}$ a 9,23 e média igual a 4,91 mg. $\mathrm{L}^{-1}$ (Tabela 2), apresentando correlação com a profundidade dos filtros de $56 \%$, com o pH de $65 \%$, temperatura de $68 \%, 77 \%$ com $\mathrm{HCO}_{3}^{-}, 78 \%$ com $\mathrm{Na}^{+}$e $84 \%$ com $\mathrm{Mg}^{2+}$. A variabilidade da concentração de sílica é devida principalmente à temperatura e $\mathrm{pH}$, cujo aumento provoca maior dissolução da sílica dos minerais (SINELLI, 1979). Silva (2005) identificou o teor médio de sílica de $11,46 \mathrm{mg} . \mathrm{L}^{-1}$ das formações Alter do Chão, Nhamundá e Solimões, valor semelhante ao encontrado neste trabalho, e relacionou às características mineralógicas locais, onde predominam 


\begin{tabular}{|l|r|}
\hline Recebido em: $18 / 05 / 2006$ & HOLOS Environment, v.7 n.1, 2007 - P. 9 \\
\hline Liberado para Publicação em: 18/01/2007 & ISSN:1519-8421 (CD-ROM) / ISSN:1519-8634 (ON-LINE) \\
\hline
\end{tabular}

rochas friáveis, pouco coesas, com intercalação de rochas mais endurecidas e restritas aos níveis de ocorrência do Arenito Manaus e de crostas lateríticas e/ou linhas-depedra (sílico ferruginosas), constituídas predominantemente por sedimentos sob a forma de folhelhos com intercalações de arenitos finos, argilas, argilitos, siltitos, arenitos e conglomerados, pobres em fosseis e à processos intempéricos intensos.

Tabela 2 - Resultados das Variáveis Analisadas nas Amostras de Água Subterrânea de Manaus Durante Período de Cheia Local.

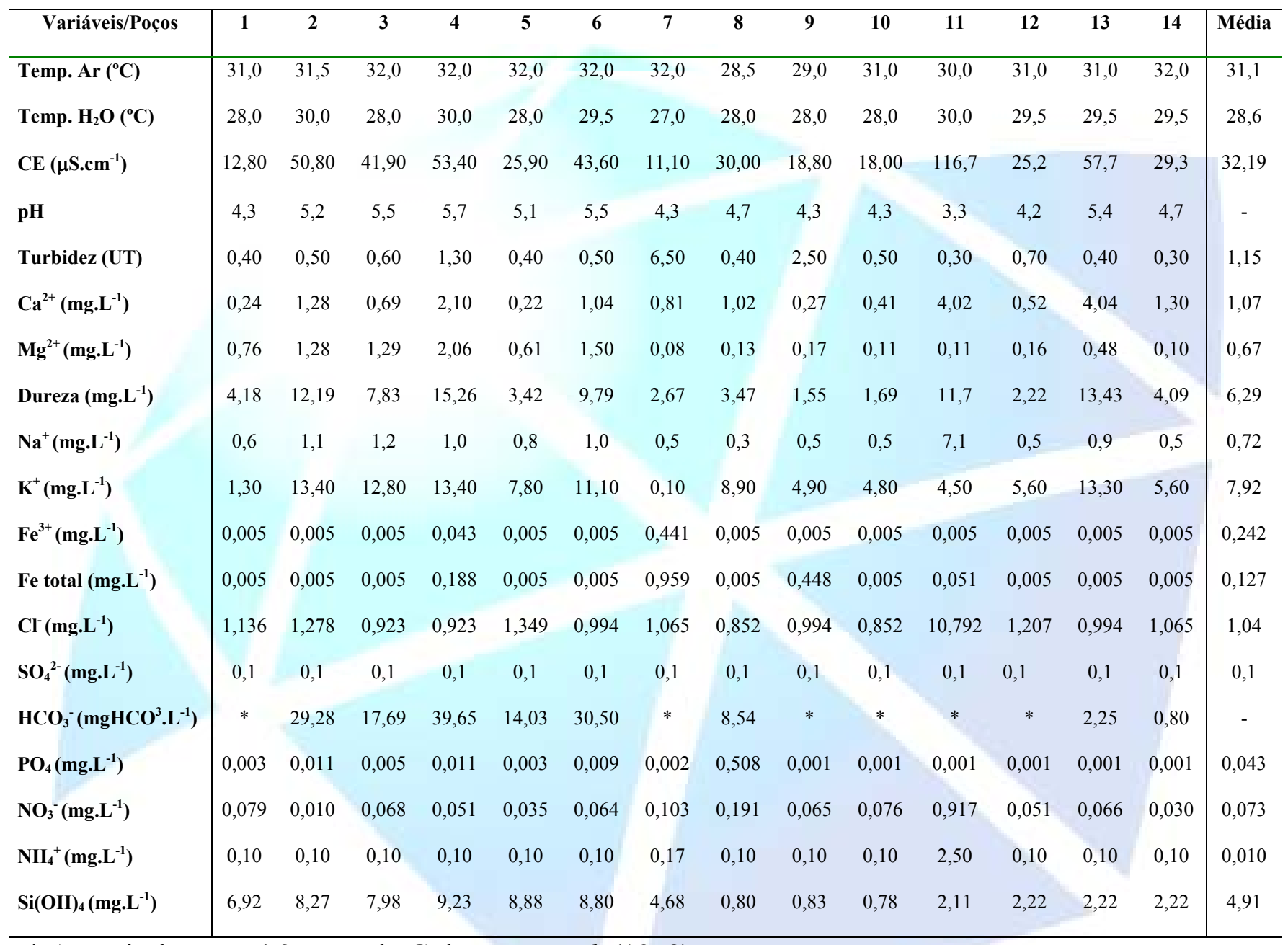

* A partir de $\mathrm{pH}>4,3$ segundo Golterman et al. (1978).

Nas águas subterrâneas estudadas, as concentrações dos cátions $\left(\mathrm{Ca}^{2+}+\mathrm{Mg}^{2+}+\mathrm{Na}^{+}+\mathrm{K}^{+}\right)$e ânions $\left(\mathrm{Cl}^{-}+\mathrm{HCO}_{3}{ }^{-}+\mathrm{SO}_{4}{ }^{2-}\right)$ foram baixas. Os poços $\mathrm{n}^{\mathrm{o}} 2,3,4$, 6,8 e 13, apresentaram os teores mais elevados e equivalência em proporções. Dentre estes, o poço $\mathrm{n}^{\mathrm{o}} 4$ foi o que apresentou os maiores valores de $\mathrm{Mg}^{2+}, \mathrm{K}^{+}$, $\mathrm{HCO}_{3}^{-}, \mathrm{SO}_{4}{ }^{2-}$ (Figura 2), $\mathrm{CE}$ e pH (Tabela 2). Porém, os poços $\mathrm{n}^{\circ} 1$ e 7 apresentaram as águas com menores concentrações catiônicas (Figura 2). 
CÁTIONS

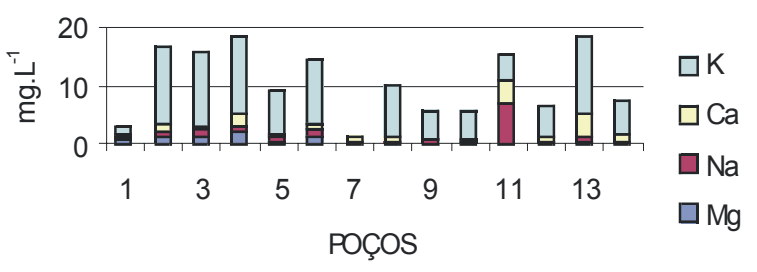

ÂNIONS

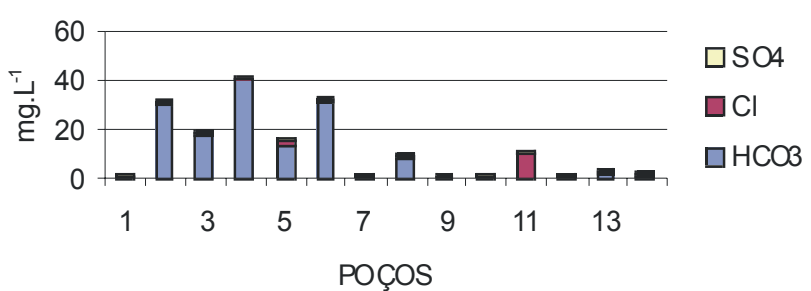

Figura 2. Distribuição das Concentrações dos Cátions $\left(\mathrm{Ca}^{2+}+\mathrm{Mg}^{2+}+\mathrm{Na}^{+}+\mathrm{K}^{+}\right)$e Ânions $\left(\mathrm{Cl}^{-}+\mathrm{HCO}_{3}^{-}+\mathrm{SO}_{4}{ }^{2-}\right)$ nas águas subterrâneas amostradas em Manaus - AM.

A direção do fluxo subterrâneo diverge, segundo dados coletados, a partir da região onde se encontram os poços $\mathrm{n}^{\mathrm{o}} 1$ e 7 (Figura 3). Silva (2005) verificou que em Manaus, a direção de fluxo subterrâneo diverge da porção Noroeste, sendo semelhante ao verificado por Silva (1999), que fez referência à direção de fluxo subterrâneo divergindo, preferencialmente, da porção Centro-Noroeste. De acordo com a CPRM (2002a), o fluxo principal das águas subterrâneas de Manaus exibe direção de NE para SW e secundários de direções diversas.

De acordo com a Figura 2, os poços com maiores concentrações de elementos catiônicos dissolvidos são os $\mathrm{n}^{\circ} 2,3,4,6,8$ e 13 com predomínio para potássio. Os poços $n^{\circ} 1$ e 7 localizam-se nas áreas com menor nível hidrostático (Figura 3) e apresentam baixas concentrações catiônicas. Quanto aos ânions dissolvidos, os poços $\mathrm{n}^{\circ} 2,3,4,5,6$ e 8 apresentaram os maiores valores aniônicos com importância para o bicarbonato (Figura 2). Então, essas características são oriundas, provavelmente, da extensa faixa de cimentação e profundidade dos poços (Tabela 1) ou pelo posicionamento desses em áreas específicas em Manaus (Figura 3).

Os mapas de isovalores de $\mathrm{NE}, \mathrm{pH}, \mathrm{CE}, \mathrm{K}^{+}, \mathrm{Mg}^{2+}, \mathrm{HCO}_{3}{ }^{-}$e $\mathrm{Si}(\mathrm{OH})_{4}$ mostram que as concentrações dos teores desses elementos aumentam de acordo com a direção do fluxo de água subterrânea para zonas onde o NE encontra-se a uma maior profundidadede (Sentido Noroeste) (Figura 3). Sendo o mesmo verificado por Silva (1999) para $\mathrm{pH}, \mathrm{CE}, \mathrm{K}^{+}, \mathrm{Mg}^{2+}, \mathrm{HCO}_{3}^{-}$.

No diagrama (Figura 4) foi possível verificar a predominância de $\mathrm{Na}^{+}+\mathrm{K}^{+}$, o teor de $\mathrm{Ca}^{2+}>\mathrm{Mg}^{2+}$ nos poços $\mathrm{n}^{\circ} 2,7,8,10,11,12,13$ e 14 , mesmo teor de $\mathrm{SO}^{2-}{ }_{4} \mathrm{e}$ aumento do $\mathrm{HCO}_{3}{ }^{-}$em relação ao verificado por Silva (1999) no período de seca.

Em relação aos demais parâmetros, constatou-se altos teores de $\mathrm{Ca}^{2+}, \mathrm{Na}^{+}, \mathrm{K}^{+}$, $\mathrm{Cl}^{-}, \mathrm{NO}_{3}{ }^{-}$e $\mathrm{NH}_{4}{ }^{+}$no poço $\mathrm{n}^{\circ} 11$, em relação aos poços amostrados (Figura 2 e 3), sendo oriundo, provavelmente, da má construção ou manutenção do poço, ou ainda de poluição antropogênica, sendo esta área densamente povoada.

Verificou-se extrema similaridade com as águas subterrâneas estudadas por Silva (1999) em período de seca local, diferindo apenas num pequeno acréscimo dos 


\begin{tabular}{|l|r|}
\hline Recebido em: $18 / 05 / 2006$ & HOLOS Environment, v.7 n.1, 2007 - P. 11 \\
\hline Liberado para Publicação em: 18/01/2007 & ISSN:1519-8421 (CD-ROM) / ISSN:1519-8634 (ON-LINE) \\
\hline
\end{tabular}

teores dos elementos, evidenciando breve infiltração dessas águas no aqüífero (Tabela 1 e Figura 4).
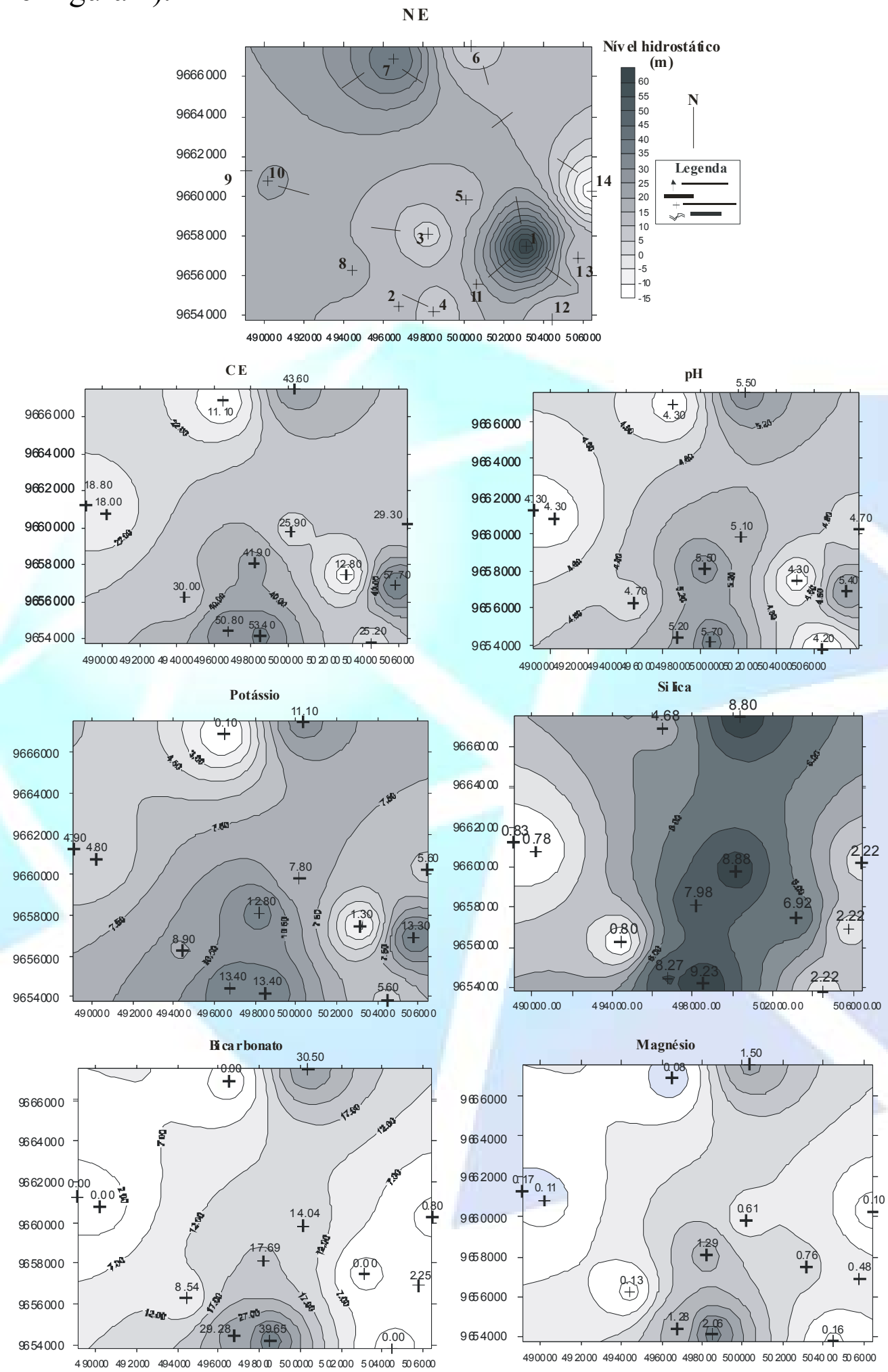

Figura 3. Mapas de Isovalores de NE, CE, pH, Potássio, Ś́lica, Bicarbonato e Magnésio Amostrados em Águas Subterrâneas em Manaus - AM. 
Quanto a hidroquímica, as águas são pouco mineralizadas e conforme a Portaria $n^{\circ} 518$ de 25/03/2004 do Ministério da Saúde, as águas estudadas são de excelente qualidade para o consumo humano e industrial, quanto às variáveis analisadas.

Estudos hidroquímicos realizados por Santos e Ribeiro (1988) e Silva e Bonotto (2000) também mostraram pureza da água de sub-superfície da região estuda, refletindo a importância do escoamento e infiltração, da alta precipitação pluviométrica, evapotranspiração e pobreza química dos materiais percolados.

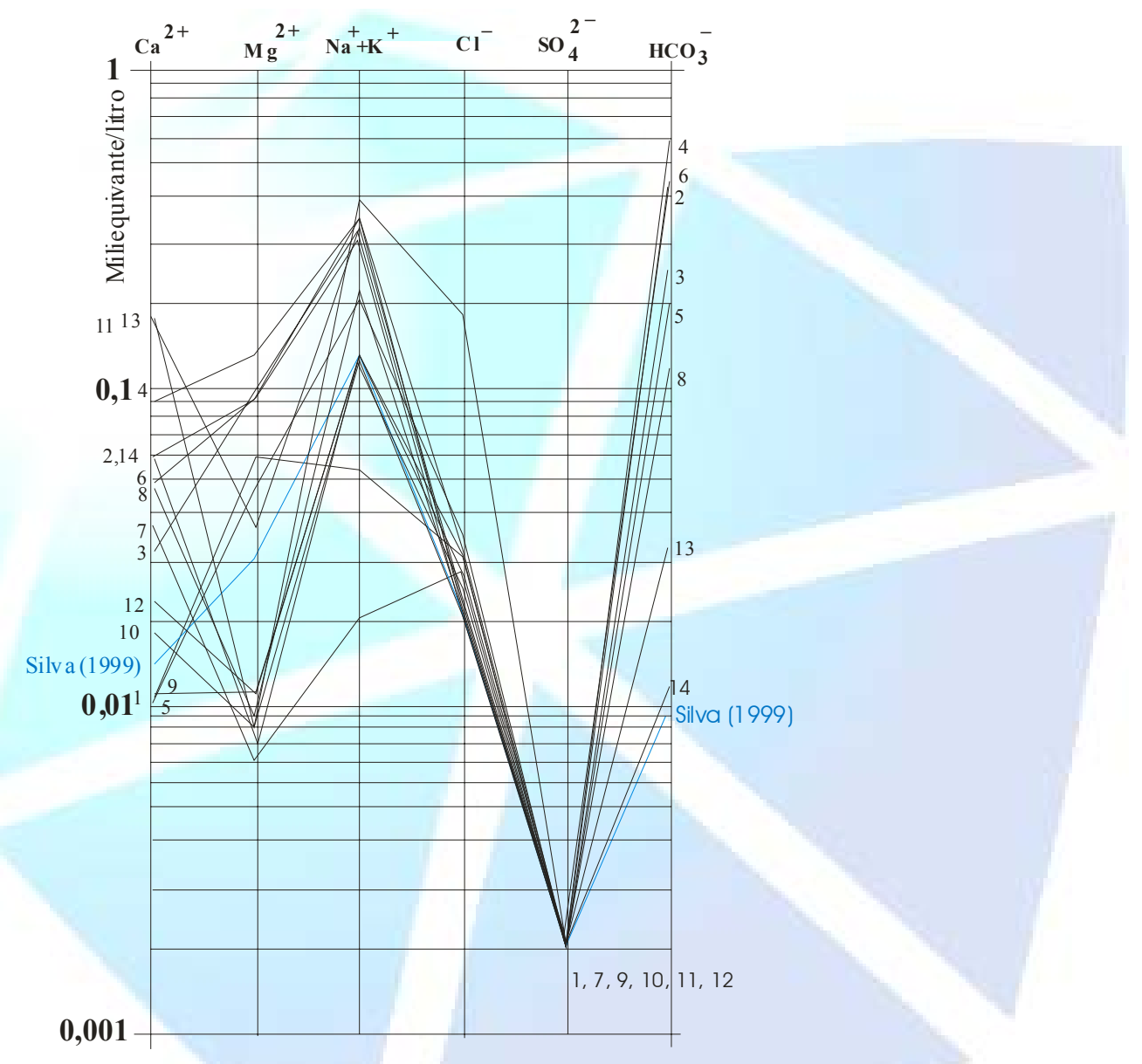

Figura 4. Diagrama de Schoeller (1962) com concentrações dos elementos estudados em águas subterrâneas em Manaus - AM (Modificado).

De acordo com o diagrama de Piper (1944), as águas amostradas em sua maioria, são potássicas, ocorrendo uma delas com caráter misto (poço $\left.\mathrm{n}^{\circ} 1\right)$ e outra cálcica (poço $\mathrm{n}^{\mathrm{o}} 7$ ). Quanto aos ânions dissolvidos, as amostras aglomeram-se no campo das cloretadas e bicarbonatadas (Figura 5). Dessa forma, as águas dos poços foram classificadas como: bicarbonatadas potássicas (poços $\mathrm{n}^{\circ} 2,3,4,5,6,8$ e 13) e Cloretadas potássicas (poços $\mathrm{n}^{\circ} 1,7,9,10,11,12$ e 14). 


\begin{tabular}{|l|r|}
\hline Recebido em: $18 / 05 / 2006$ & HOLOS Environment, v.7 n.1, 2007 - P. 13 \\
\hline Liberado para Publicação em: 18/01/2007 & ISSN:1519-8421 (CD-ROM) / ISSN:1519-8634 (ON-LINE) \\
\hline
\end{tabular}

No diagrama (Figura 5), a área hachurada representa o posicionamento das amostras coletadas por Silva (1999) no período de seca local. Observa-se, então, o ganho de $\mathrm{Ca}^{2+}, \mathrm{Cl}^{-}$e $\mathrm{HCO}_{3}^{-}$no período de cheia. Evidenciando a importante contribuição da infiltração dessas águas para o aqüífero, pois para todos os poços amostrados, a cimentação teve profundidade considerável (Tabela 1).

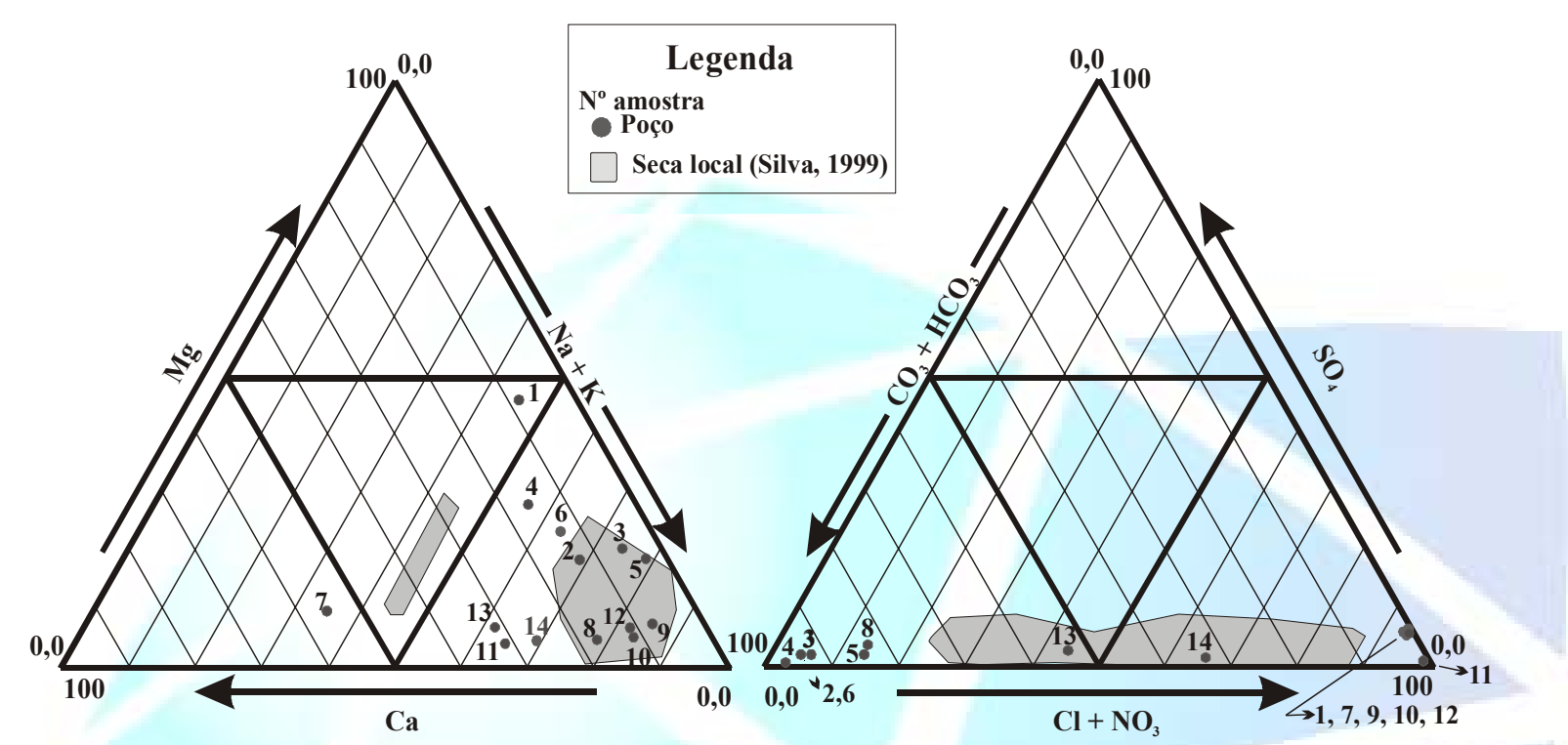

Figura 5. Classificação das Águas Subterrâneas de Manaus de Acordo com o Diagrama de Piper (1944) (Modificado).

\section{CONCLUSÃO}

Através do desenvolvimento deste trabalho, foi possível observar que houve pequeno acréscimo nos teores dos elementos dissolvidos nas águas subterrâneas em Manaus, no período de cheia local;

Considerando-se os valores médios obtidos para os principais cátions e ânions dissolvidos, as águas dos poços amostrados foram classificadas como: bicarbonatadas potássicas e cloretadas potássicas;

De acordo com as variáveis analisadas, a qualidade das águas subterrâneas é apropriada para o consumo humano e industrial (com algumas restrições ao $\mathrm{pH}$ ácido), uma vez que foram determinadas concentrações baixas dos constituintes dissolvidos, inserindo-se todos eles no contexto dos padrões de potabilidade estabelecidos pela Portaria ${ }^{\circ} 518$ de 25/03/2004 do Ministério da Saúde.

Em Manaus, existem milhares de poços tubulares e escavados de baixo rendimento, explorando o aqüífero no nível freático mais elevado, oferecendo abastecimento não tratado, sem monitoração e proteção sanitária na maioria dos casos, gerando potenciais focos de contaminação. Portanto, devido às características 
ambientais, geológicas, hidrogeológicas e uso potencial das águas subterrâneas, devese exercer proteção geral do aqüífero, com medidas de controle das atividades locais, construção de poços com adequação às normas técnicas, fiscalização na exploração dessas águas, assim como, promoverem educação e conscientização ambiental para preservação do meio ambiente como um todo.

\section{REFERÊNCIAS}

APHA. AMERICAN PUBLIC HEALTH ASSOCIATION. Standard methods for the examination of water and wastewater. $16 \mathrm{ed}$. Washington: APHA, 1985. 1269p.

CUNHA, P.R.C.; GONZAGA, F.G.; COUTINHO, L.F.C.; FEIJÓ, F.J. Bacia do Amazonas. Bol. Geocienc., Rio de Janeiro, v.8, p.47-55, 1994.

DAMIÃO, R.N.; SOUZA, M.M.; MEDEIROS, M.F. Projeto argila Manaus. Manaus: DNPM/CPRM, 1972. 65p. Relatório interno.

DAEMON, R.F. Contribuição à datação da Formação Alter do Chão, Bacia do Amazonas. Geociências, São Paulo, v.5, n.2, p.78-84, 1975.

FERNANDES FILHO, L.A., COSTA, M.L.,COSTA, J.B.S. Registros neotectônicos nos lateritos de Manaus-AM. Geociências, São Paulo, v.16, p. 9-33, 1997.

FORTI, M.C.; MOREIRA-NORDEMANN, L.M. Rainwater and throughfall chemistry in a "terra firme" rain forest: Central Amazonia. Geophys. Res., v.96, p.7415-21, 1991.

GOLTERMAN, H.; CLYMO, R.S.; OHMSTAD,.M.A.M. Methods for physical \& chemical analysis of freshwater. Oxford: Blackwell Scientific Publications, 1978. 213p. (Handbook n. 8).

IBGE. INSTITUTO BRASILEIRO DE GEOGRAFIA E ESTATÍSTICA. Disponível

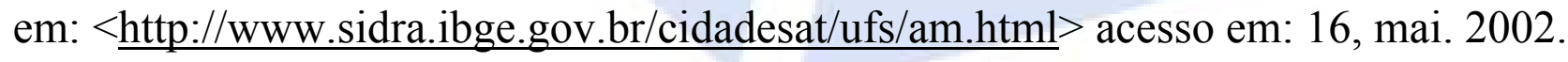

MORAES, E.L.; COSTA, M.L. O caulim de Manaus-Itacoatiara: aspectos mineralógicos e geoquímicos. Geociências, São Paulo, v.16, p. 35-53, 1997.

PETRI, S.; FÚlFARO, V.J. Geologia do Brasil: fanerozóico. São Paulo: Edusp, 1988. v.9, 631p.

PIPER, A.M. A graphic procedure in the geochemical interpretation of wateranalyses. Trans. Amer. Geophysical Union, USA, v.25, p.914-28, 1944. 


\begin{tabular}{|l|r|}
\hline Recebido em: $18 / 05 / 2006$ & HOLOS Environment, v.7 n.1, 2007 - P. 15 \\
\hline Liberado para Publicação em: 18/01/2007 & ISSN:1519-8421 (CD-ROM) / ISSN:1519-8634 (ON-LINE) \\
\hline
\end{tabular}

PRANCE, G.T.; LOVEJOY, T.E. Amazônia: key environments. Oxford: Pergamon Press, 1985. p.400.

REBOUÇAS, A.C. Panorama da degradação do ar, da água doce e da terra no Brasil. São Paulo: IEA/USP; Rio de Janeiro: Academia Brasileira de Ciências, 1997. 150 p.

SANTOS, A.C. Noções de hidroquímica. In: Hidrogeologia: conceitos e aplicações. 2 ed. Fortaleza: CPRM/REFO, LABHID-UFPE, 2000. p.81-108.

SANTOS, U.M; RIBEIRO, M.N.G. A hidroquímica do rio Solimões-Amazonas. Acta Amaz., Manaus, v.18, p. 145-72, 1988.

SAWYER C.N. e MCCARTY, P.L. Chemistry for sanitary engineers. New York: McGraw-Hill, 1967. 535p.

SCHOELLER, H. Les eaux souterraines. Paris: Masson \& Cie., 1962. 642p.

SILVA, M.L. Hidroquímica elementar e dos isótopos de urânio no aqüífero de Manaus - AM. Rio Claro: UNESP, 1999. 82p. Dissertação (Mestrado em Conservação e Manejo de Recursos), Centro de Estudos Ambientais, Universidade Estadual Paulista, 1999.

SILVA, M. L. Estudo hidroquímico e dos isótopos de urânio nas águas subterrâneas em cidades do estado do Amazonas (AM). Rio Claro: UNESP, 2005. 200p. Tese (Doutorado em Geociências e Meio Ambiente), Instituto de Geociências e Ciências Exatas, Universidade Estadual Paulista, 2005.

SILVA, M.S.R. Metais pesados em sedimentos de fundo de igarapés (Manaus-AM). Belém, 1996. 120p. Dissertação (Mestrado Geoquímica Ambiental), Universidade Federal do Pará, 1996.

SILVA, M.L. e BONOTTO, D.M. Caracterização hidrogeoquímica na Formação Alter do Chão, Município de Manaus (AM). $1^{\text {st }}$ Joint World Congress on Groundwater. Fortaleza, 2000.

SINELLI, O. Química das águas subterrâneas: Aplicação a águas subterrâneas do Brasil Meridional. Ribeirão Preto: USP, 1979. 130p. Tese (Livre Docência), Faculdade de Filosofia, Ciências e Letras de Ribeirão Preto, 1979.

STRICKLAND, J.D.H.; PARSONS, T.R. A manual of sea water analysis. Bull. Fish Res. Bd. Can., Canada, v.125, p. 1-185, 1968. 